\title{
REVIEW
}

For reprint orders, please contact: reprints@futuremedicine.com

\section{Sorafenib for the treatment of hepatocellular carcinoma}

\author{
Marcus Alexander Wörns*1 \& Peter Robert Galle
}

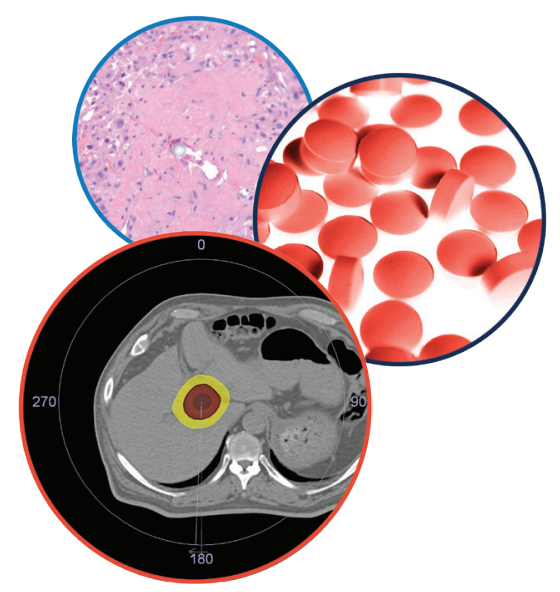

Sorafenib is an oral multikinase inhibitor with antiproliferative and antiangiogenic properties that targets
various types of intracellular serine/threonine (Raf-1 and BRAF) and cell surface receptor tyrosine kinases
(including VEGFR-2/-3, PDGFR- $\beta$, c-KIT, FLT3 and RET).
Sorafenib is approved for the treatment of patients with (unresectable) hepatocellular carcinoma and
has been established as the standard of care in Child-Pugh A patients with advanced hepatocellular
therapies in the intermediate stage of the disease (Barcelona Clinic Liver Cancer B).
Several baseline characteristics could be identified as negative prognostic indicators for the overall
survival, first and foremost impaired liver function. Treatment of Child-Pugh B patients might be feasible
in clinical practice; however, the survival benefit remains questionable.
If patients achieve ongoing clinical benefit from sorafenib treatment in clinical practice, treatment should
be discontinued only in case of clear radiological tumor progression according to modified Response
Evaluation Criteria In Solid Tumors.
Most common adverse events (AEs) are diarrhea, hand-foot skin reaction and fatigue, and, to lesser
extent, arterial hypertension, rash, dry skin and weight loss.
AEs are manageable in most cases in daily practice with careful patient education, preventive measures
and early diagnosis.
Most grade $1 / 2$ AEs can be relieved by symptomatic treatment; however, grade 3/4 AEs may require
temporary dose reduction or treatment interruption, or in severe or persistent cases, permanent
discontinuation.
In case of persistent AEs, patients may be treated with a permanent dose reduction instead of
lower doses of sorafenib might retain anticancer activity.
a

'First Department of Internal Medicine, University Medical Center of the Johannes Gutenberg University, Langenbeckstrasse 1, 55131 
SUMMARY The multikinase inhibitor sorafenib, which inhibits targets related to tumor cell proliferation and angiogenesis, was the first systemic agent to demonstrate a significant improvement in the overall survival for patients with advanced hepatocellular carcinoma (HCC) in two large randomized controlled Phase III trials. Together with its manageable safety profile (mainly diarrhea, hand-foot skin reaction and fatigue), sorafenib was approved for the treatment of patients with (unresectable) HCC in 2007. Since then, sorafenib has been established as the standard of care in Child-Pugh A patients with advanced HCC or in those ineligible for or after failure of locoregional therapies in the intermediate stage of the disease. This article summarizes current knowledge and future perspectives regarding the use of sorafenib in patients with HCC.

Hepatocellular carcinoma (HCC) is the third most common cause of cancer-related death [1] and the leading cause of death among patients with liver cirrhosis [2]. Globally, the majority of cases occur due to endemic hepatitis $B$ virus (HBV) infection; however, in western countries and Japan, the incidence of $\mathrm{HCC}$ will further rise due to the burden of hepatitis $\mathrm{C}$ virus (HCV) infection and the increasing incidence of nonalcoholic steatohepatitis [3]. Despite progress in surveillance programs, potentially curative treatment approaches (resection, liver transplantation or local ablation) can be offered to only a small proportion of patients $[3,4]$ in the early stage of the disease (Barcelona Clinic Liver Cancer [BCLC] class 0/A) according to the BCLC classification [5]. In the intermediate stage (BCLC B; asymptomatic patients, multifocal tumors without macrovascular invasion [MVI] or extrahepatic spread [EHS]) transarterial chemoembolization (TACE) can provide survival benefits $[3,6,7]$. However, due to late diagnosis and underlying liver cirrhosis, most patients are diagnosed in an advanced (BCLC C) or even terminal stage (BCLC D) with limited treatment options. After years of therapeutic nihilism due to inefficacy of conventional cytotoxic chemotherapy and other compounds [8], the increased knowledge of molecular hepatocarcinogenesis today provides an opportunity for molecularly targeted therapy (MTT) [9]. Although HCC is a heterogeneous tumor with not yet identified oncogene addiction, several signaling pathways and cell surface receptors mainly related to cell proliferation and angiogenesis, such as the ubiquitous Ras/Raf/MAPK/MEK/ ERK pathway or VEGFR, have been repeatedly identified as crucial for hepatocarcinogenesis [10]. Positive data from two pivotal Phase III trials, SHARP [11] and the Asia Pacific trial [12], assessing the safety and efficacy of the oral multikinase inhibitor sorafenib (BAY43-9006, Nexavar ${ }^{\circledR}$, Bayer HealthCare Pharmaceuticals, Leverkusen, Germany) broadened the horizon for patients with advanced disease. Sorafenib blocks tumor cell proliferation and angiogenesis by targeting several serine/threonine and receptor tyrosine kinases (RTKs) such as Raf-1 and VEGFR-2 [13]. Sorafenib was the first systemic agent to demonstrate a statistically significant improvement in the overall survival (OS) for patients with advanced HCC and well-preserved liver function Child-Pugh (CP) A, and the US FDA and EMA approved sorafenib for the treatment of patients with (unresectable) HCC in 2007.

\section{Body of review}

\section{- Information resource}

Studies for consideration in this article were retrieved from the MEDLINE database using the search terms "HCC" and "sorafenib". In addition, relevant congress abstracts were included in this review. Further information regarding sorafenib can be found in the Nexavar product information on the EMA homepage [14]. The search included literature published in English up to August 2013.

\section{- Overview of the market}

Although the advent of sorafenib represented a major breakthrough and confirmed the effectiveness of MTT in this therapeutic area of unmet need, it was just the first step towards an improved systemic therapy in HCC [15]. The mechanism of action is poorly understood with a disease stabilization lasting only several months, most probably due to acquired resistance. Median OS in clinical practice does not exceed 1 year in most cases and relevant toxicities can negatively affect the tolerability and efficacy of sorafenib. In contrast with the Phase III trials, both the FDA and EMA indications do not limit the prescription of sorafenib to CP A patients; however, until recently there was only a preliminary picture of the use of sorafenib in patients with more impaired liver function (CP B/C). Baseline factors and biomarkers 
predicting response and prognosis on sorafenib treatment are not well defined and the radiological evaluation of tumor response remains challenging $[16,17]$, because sorafenib rarely produces tumor shrinkage resulting in a low objective response rate (ORR) according to conventional Response Criteria in Solid Tumors (RECIST). These findings underscore the need to optimize the outcome of patients treated with sorafenib in clinical practice and indicate the rationale for the investigation of synergistic combinations and alternative agents. Sorafenib was tested in multiple trials in advanced HCC during the last few years, either head to head, or in combination with novel agents and conventional chemotherapeutics [15]. However, no other targeted agent or regimen has proven efficacy to significantly improve the survival of patients with advanced HCC in a Phase III trial (e.g., sunitinib [18], linifanib [19], brivanib [20,21] and erlotinib [22]) in the first- and second-line setting so far and no standard treatment exists for patients with acquired resistance or intolerance to sorafenib. Sorafenib is also under investigation in the earlier stages of disease in the adjuvant setting after potentially curative approaches and there is a rationale to combine TACE with sorafenib in the intermediate stage of the disease to inhibit the neoangiogenic activity caused by ischemic injury following TACE. However, the clinical implication of these approaches has to be defined.

\section{- Introduction to the compound}

Sorafenib is approved for the treatment of patients with HCC (EMA) or unresectable HCC (FDA); however, in clinical practice, sorafenib is endorsed by the European Association for the Study of the Liver [3] and the American Association for the Study of Liver Diseases [7] mainly for patients with advanced HCC (BCLC C) or in those ineligible for or after failure of locoregional therapies in the intermediate stage of the disease (BCLC B).

\section{- Chemistry}

The following information is taken from the Nexavar product information, which can be found on the EMA homepage [14]. One tablet of Nexavar contains $274 \mathrm{~g}$ of sorafenib tosylate (BAY 43-9006), equivalent to $200 \mathrm{mg}$ of sorafenib. Sorafenib tosylate has the chemical name 4-(4-[3-(4-chloro-3 [trifluoromethyl]-phenyl)ureido]phenoxy)
N2-methylpyridine-2-carboxamide 4-methylbenzene-sulfonate. Sorafenib tosylate has a molecular weight of $637.0 \mathrm{~g} / \mathrm{M}$ and is practically insoluble in aqueous media, slightly soluble in ethanol and soluble in polyethylene glycol 400 .

\section{- Pharmacodynamics}

The biaryl urea sorafenib is a multikinase inhibitor that has demonstrated both anti-proliferative and -angiogenic properties by targeting various types of intracellular serine/threonine and cell surface RTKs. According to in vitro studies and preclinical xenograft models of different tumor types, sorafenib inhibits several members of the MAPK signaling pathway, including Raf1(c-Raf), wild-type BRAF and V600E-mutant BRAF (Table 1) [13,23,24]. In addition, sorafenib exerts its anticancer properties by inhibiting multiple proangiogenic RTKs, including VEGFR-1/-2/-3, PDGFR- $\beta$, c-KIT, Flt-3 and the RET tyrosine kinase. Sorafenib also demonstrated proapoptotic effects by enhancing proteasomal degradation of the antiapoptotic Mcl-1 protein in several cancer cell lines [25-27]. Besides its direct effect on tumor cells, sorafenib may additionally have an immune stimulatory function by triggering proinflammatory activity of macrophages and subsequently inducing anti-tumor natural killer cell responses [28].

\section{- Pharmacokinetics \& metabolism}

The following information is taken from the Nexavar product information which can be found on the EMA homepage [14]. The recommended daily dose of sorafenib is $400 \mathrm{mg}$ $(2 \times 200 \mathrm{mg}$ tablets) taken twice daily without food (at least $1 \mathrm{~h}$ before or $2 \mathrm{~h}$ after a meal). After administration of sorafenib the mean relative bioavailability is $38-49 \%$ when compared with an oral solution. Following oral administration sorafenib reaches peak plasma concentrations in approximately $3 \mathrm{~h}$. When given with a high-fat meal, sorafenib absorption was reduced by $30 \%$ compared with administration in the fasted state. Blood $\mathrm{C}_{\text {max }}$ and area under the curve (AUC) increased less than proportionally beyond doses of $400 \mathrm{mg}$ administered twice daily. In vitro binding of sorafenib to human plasma proteins is $99.5 \%$. Multiple dosing of sorafenib for 7 days resulted in a 2.5- to 7-fold accumulation compared with single-dose administration. Steady-state plasma sorafenib concentrations are achieved within 7 days, with a peak to trough ratio of mean 


\begin{tabular}{|c|c|}
\hline Target & $\mathrm{IC}_{50}(\mathrm{nmol} / \mathrm{I})$ \\
\hline Raf-1 & 6 \\
\hline BRAF wild-type & 22 \\
\hline V600E mutant BRAF & 38 \\
\hline VEGFR-2 & 90 \\
\hline Murine VEGFR-2 & 15 \\
\hline Murine VEGFR-3 & 20 \\
\hline Murine PDGFR- $\beta$ & 57 \\
\hline Flt-3 & 58 \\
\hline c-KIT & 68 \\
\hline RET & 47 \\
\hline FGFR-1 & 580 \\
\hline \multicolumn{2}{|l|}{ Data taken from $[13,23,24]$. } \\
\hline
\end{tabular}

concentrations of less than 2 . The elimination half-life of sorafenib is approximately $25-48 \mathrm{~h}$. Sorafenib is metabolized primarily in the liver and undergoes oxidative metabolism, mediated by CYP3A4, as well as glucuronidation mediated by UGT1A9. Sorafenib conjugates may be cleaved in the GI tract by bacterial glucuronidase activity, allowing reabsorption of unconjugated drug. Sorafenib accounts for approximately $70-85 \%$ of the circulating analytes in plasma at steady state. Eight metabolites of sorafenib have been identified, of which five have been detected in plasma. The main circulating metabolite of sorafenib in plasma, the pyridine $\mathrm{N}$-oxide, shows in vitro potency similar to that of sorafenib. This metabolite comprises approximately $9-16 \%$ of circulating analytes at steady state. Following oral administration of a 100 $\mathrm{mg}$ dose of a solution formulation of sorafenib, $96 \%$ of the dose was recovered within 14 days, with $77 \%$ of the dose excreted in the feces, and $19 \%$ of the dose excreted in the urine as glucuronidated metabolites. Unchanged sorafenib, accounting for $51 \%$ of the dose, was found in the feces but not in the urine. Analyses of demographic data suggest that there is no relationship between pharmacokinetics (PKs) and age (up to 65 years), gender or bodyweight. There are no clinically relevant differences in PKs between Caucasian and Asian subjects. No dose adjustment is required in patients with mild, moderate or severe renal impairment. No dose adjustment is required in patients with $\mathrm{CP} \mathrm{A}$ or $\mathrm{B}$ (mild to moderate) hepatic impairment. Administration of rifampicin for 5 days before administration of a single dose of sorafenib resulted in an average
$37 \%$ reduction of sorafenib AUC. Other inducers of CYP3A4 activity and/or glucuronidation (e.g., Hypericum perforatum, also known as $\mathrm{St}$ John's wort, phenytoin, carbamazepine, phenobarbital and dexamethasone) may also increase metabolism of sorafenib and, thus, decrease sorafenib concentrations. Sorafenib exposure decreases when coadministered with neomycin. Coadministration of capecitabine and sorafenib resulted in no significant change in sorafenib exposure, but a $15-50 \%$ increase in capecitabine exposure. Coadministration of doxorubicin and sorafenib resulted in a $21 \%$ increase in the AUC of doxorubicin.

\section{- Clinical efficacy \\ Phase I}

Sorafenib has been investigated as monotherapy in four Phase I trials in patients with advanced refractory solid tumors including HCC [29], each following a dose-escalation procedure and a different treatment schedule (including continuous dosing) (Box 1). Sorafenib was generally well tolerated, particularly at doses $\leq 400 \mathrm{mg}$ twice daily, and the majority of adverse events (AEs) were mild to moderate in severity and easily manageable. The most frequently reported drug-related AEs according to the Common Terminology Criteria of Adverse Events at any grade included fatigue (40\%), anorexia (35\%), diarrhea (34\%), rash/desquamation (27\%) and hand-foot skin reaction (HFSR) (25\%). The most frequent drug-related AEs of grade $\geq 3$ in severity were HFSR (8\%), fatigue (6\%) and diarrhea (4\%). The maximum tolerated dose (MTD) was defined as $400 \mathrm{mg}$ twice daily continuously in three of the four trials. Sorafenib demonstrated preliminary anti-tumor activity primarily by inducing disease stabilization and particularly among patients with renal cell carcinoma (RCC) and HCC. Based on these findings, continuous sorafenib $400 \mathrm{mg}$ twice daily was chosen as the optimal regimen for Phase II/III studies.

Sorafenib (200-400 mg twice daily) was tested in a Phase I study in 27 Japanese patients with unresectable HCC and liver function $\mathrm{CP} A / \mathrm{B}$ (Box 1) [30]. There were no clinically relevant differences in PKs between CP A and B patients, and according to authors opinion no major differences in the incidence and grade of drug-related AEs. However, the differences at the $400-\mathrm{mg}$ dose level were diarrhea (CP A 62.5 vs CP B 33.3\%), weight loss (50.0 vs 
Box 1. Summary of Phase I and II trial results in the development of sorafenib monotherapy in hepatocellular carcinoma including safety and tolerability.

Phase I

- Strumberg et al. [29]

- MTD: 400 mg twice daily

- Most frequent drug-related AEs of grade $\geq 3$ : HFSR (8\%), fatigue (6\%) and diarrhea (4\%)

- Furuse et al. [30]

- Incidence and grade of drug-related AEs, no major differences between CP A and B patients

- Level of response (\%):

- Complete: 0

- Partial: 3.7

- Stable: 77.8

- Progressive: 11.1

- DCR: 81.5

- Miller et al. [31]

- Empiric starting doses $200 \mathrm{mg}$ twice daily in patients with bilirubin more than 1.5- to $\leq 3$-times ULN; not even 200 mg every third day in patients with bilirubin more than three- to ten-times ULN. Patients with mild-to-moderate renal dysfunction (creatinine clearance $40-59 \mathrm{ml} / \mathrm{min}$ ) may start with $400 \mathrm{mg}$ twice daily

Phase II

- Abou-Alfa et al. [32,33]

- Median sorafenib treatment time: 3.4 months

- Level of response (\%):

- Complete: 0

- Partial: 2.2

- Minor response: 5.8

- Stable: 33.6

- Progressive: 35.0

- DCR: 41.6

- Median TTP (investigator assessment): 5.5 (4.2) months

- Median OS: 9.2 months

- Most frequent drug-related AEs of grade $\geq 3$ : fatigue (9.5\%), diarrhea (8\%) and HFSR (5.1\%)

- Median sorafenib treatment time (CP A/CP B): 4.0 months/1.8 months

- Median PFS (CP A/CP B): 4.4 months/2.5 months

- Median TTP (CP A/CP B): 5.0 months/3.0 months

- Median OS (CP A/CP B): 9.5 months/3.2 months

- Yau et al. [34]

- Median sorafenib treatment time: 3.0 months

- Level of response (\%):

- Complete: 0

- Partial: 8

- Stable: 18.0

- Progressive: 74.0

- DCR: 26.0

- Median PFS: 3.0 months

- Median OS: 5.0 months

- Most frequent drug-related AEs of grade $\geq 3$ : diarrhea (20\%), HFSR (16\%) and thrombocytopenia $(10 \%)$

- Median OS (CP A/CP B): 5.5 months/5.0 months

- Liver function impairment (CP A/CP B): 56\%/73\%

AE: Adverse event; CP: Child-Pugh; DCR: Disease control rate; HCC: Hepatocellular carcinoma; HFSR: Hand-foot skin reaction; MTD: Maximum tolerated dose; OS: Overall survival; PFS: Progression-free survival; TTP: Time to progression; ULN: Upper limit of normal. 
16.7\%), hypertension ( 37.5 vs $16.7 \%$ ), dry skin (37.5 vs $0 \%)$ and fatigue (25.0 vs $0 \%)$. One patient $(3.7 \%)$ achieved a partial response (PR), 21 patients $(77.8 \%)$ had stable disease (SD) and three patients $(11.1 \%)$ had progressive disease (PD) according to RECIST.

Another Phase I trial sought to characterize the PKs and determine a tolerable dose of sorafenib in patients with hepatic or renal dysfunction (Box 1) [31]. Whereas the majority of patients with renal dysfunction were assessable, many patients with hepatic dysfunction were unable to complete the first 3 weeks of continuous dosing. The authors recommended the empiric starting doses of $200 \mathrm{mg}$ twice daily in patients with bilirubin ranging from 1.5- to 3-times the upper limit of normal (ULN) and not even $200 \mathrm{mg}$ every third day in patients with bilirubin more than three- to ten-times the ULN. Patients with mild-to-moderate renal dysfunction (creatinine clearance: $40-59 \mathrm{ml} / \mathrm{min}$ ) may start with $400 \mathrm{mg}$ twice daily.

\section{Phase II}

In a Phase II trial, 137 patients with advanced HCC (CP A/B, Eastern Cooperative Oncology Group [ECOG] performance status [PS] 0/1 and no prior systemic therapy) were treated with sorafenib $400 \mathrm{mg}$ twice daily (Box 1) [32]. PKs were measured in plasma samples, and biomarker analysis included immunohistochemistry of pERK in pretreatment biopsies. Median duration of sorafenib treatment was 3.4 months. On the basis of independent assessment, sorafenib induced $\mathrm{PR}$ in three patients $(2.2 \%)$, minor response in eight patients $(5.8 \%), \mathrm{SD}$ in 46 patients (33.6\%) and PD in 48 patients (35.0\%) according to modified WHO criteria. Median time to progression (TTP) was 4.2 months (investigator assessment) or 5.5 months (independent assessment), and median OS was 9.2 months, respectively. Grade 3 drug-related AEs included fatigue (9.5\%), diarrhea (8\%) and HFSR (5.1\%). Patients with higher pERK staining had a significantly longer TTP (178 vs 46 days; $\mathrm{p}=0.00034)$, suggesting $\mathrm{pERK}$ as a potential marker of response. An exploratory subanalysis evaluated the safety and efficacy of sorafenib in CP A (72\%) versus CP B (28\%) patients [33]. Median duration of sorafenib treatment ( 4.0 vs 1.8 months), median progression-free survival (PFS; 4.4 vs 2.5 months), median TTP (5.0 vs 3.0 months) and median OS (9.5 vs 3.2 months) were substantially better in CP A patients compared with CP B patients. There were no meaningful PK differences between CP A and $B$ patients; however, certain grade 3/4 AEs were more frequent in patients with CP B liver function, such as hyperbilirubinemia (53 vs $14 \%$ ), ascites (5 vs 3\%) and encephalopathy (13 vs 3\%).

Another Phase II study with sorafenib (400 mg twice daily) in patients with advanced $\mathrm{HCC}$ was conducted in an HBV-endemic Asian population (Box 1) [34]. In total, 51 patients (71\% CP A, 69\% ECOG 0, 90\% HBV-related HCC, 47\% MVI and 47\% EHS) were enrolled. The median duration of treatment was 3.0 months, and 17 patients $(33 \%)$ had the dose reduced by half due to drug-related toxicities. Four patients (8\%) achieved PR, nine patients (18\%) had SD, 38 patients $(74 \%)$ had $\mathrm{PD}$, and median PFS and OS were 3.0 and 5.0 months, respectively. Patients without EHS, particularly without lung metastasis, were more likely to benefit from sorafenib treatment $(\mathrm{p}<0.01)$. The most common toxicities were diarrhea (67\%), malaise (55\%) and HFSR (54\%). Most common grade $3 / 4$ AEs were diarrhea (20\%), HFSR (16\%) and thrombocytopenia (10\%). There were no significant differences between CP A and B patients in terms of OS (5.5 vs 5.0 months; $\mathrm{p}=0.24$ ) and drug-related toxicities (liver function impairment: 56 vs $73 \%$; $\mathrm{p}=0.236)$.

\section{Phase III}

The randomized, double-blind, placebo-controlled, Phase III SHARP trial was mainly conducted in western countries to further assess the efficacy and safety of sorafenib $400 \mathrm{mg}$ twice daily in 602 patients with advanced HCC (95\% CP A, 82\% BCLC C, 38\% MVI and 51\% EHS) and no prior systemic therapy [11] (Table 2). The median duration of treatment was 5.3 months (range: $0.2-16.1$ ) in the sorafenib group and 4.3 months (range: 0.1-16.6) in the placebo group. Overall, 227 patients in the sorafenib group $(76 \%)$ and 284 in the placebo group (94\%) received more than $80 \%$ of the planned daily dose of the study drug. The median OS was significantly longer in the sorafenib group than in the placebo group (10.7 vs 7.9 months; hazard ratio [HR]: 0.69 ; 95\% CI: $0.55-0.87$; $\mathrm{p}<0.001$ ) with survival rates at 1 year of $44 \%$ in the sorafenib group and $33 \%$ in the placebo group. The median time to symptomatic progression did not differ significantly between both groups (4.1 and 4.9 months; HR: 1.08; 
Table 2. Summary of Phase III trial results in the development of sorafenib monotherapy in hepatocellular carcinoma including safety and tolerability.

\begin{tabular}{|c|c|c|c|c|}
\hline \multirow[t]{2}{*}{ Measure } & \multicolumn{2}{|c|}{ SHARP trial (Llovet et al.) [11] } & \multicolumn{2}{|c|}{ Asia-Pacific trial (Cheng et al.) [12] } \\
\hline & Sorafenib group & Placebo group & Sorafenib group & Placebo group \\
\hline Median TTP (months) & 5.5 & 2.8 & 2.8 & 1.4 \\
\hline Median TTSP (months) & 4.1 & 4.9 & 3.5 & 3.4 \\
\hline Median OS (months) & 10.7 & 7.9 & 6.5 & 4.2 \\
\hline $\begin{array}{l}\text { Rate of discontinuation due } \\
\text { to AEs (\%) }\end{array}$ & 38 & 37 & 19.5 & 13.3 \\
\hline \multicolumn{5}{|l|}{ Level of response (\%) } \\
\hline Complete & 0 & 0 & 0 & 0 \\
\hline Partial & 2 & 1 & 3.5 & 1.3 \\
\hline Stable & 71 & 67 & 54 & 27.6 \\
\hline \multicolumn{5}{|c|}{ Most frequent drug-related AEs of grade $\geq 3$ (\%) } \\
\hline Diarrhea & 8 & 2 & 6 & 0 \\
\hline HFSR & 8 & $<1$ & 10.7 & 0 \\
\hline Fatigue & 4 & $<4$ & 3.4 & 1.3 \\
\hline
\end{tabular}

95\% CI: 0.88-1.31; $\mathrm{p}=0.77)$; however, the median TTP was significantly longer in the sorafenib group (5.5 vs 2.8 months; HR: 0.58 95\% CI: $0.45-0.74 ; \mathrm{p}<0.001)$. In the sorafenib group, seven patients $(2 \%)$ had a PR and 211 patients $(71 \%)$ had SD, whereas in the placebo group two patients (1\%) had a PR and 204 patients $(67 \%)$ had SD. The overall incidence of treatment-related AEs was $80 \%$ in the sorafenib group and $52 \%$ in the placebo group. Grade 3 drug-related AEs more common in the sorafenib arm included diarrhea (8 vs $2 \%$; $\mathrm{p}<0.001)$, HFSR (8 vs $<1 \%$; $\mathrm{p}<0.001)$, hypertension ( $2 \mathrm{vs}<1 \%$; $\mathrm{p}=0.28)$, abdominal pain $(2$ vs $1 \%$; $\mathrm{p}=0.17$ ), hypophosphatemia (11 vs $2 \%$; $\mathrm{p}<0.001)$ and thrombocytopenia (4 vs $<1 \%$; $\mathrm{p}=0.006)$. However, the rate of discontinuation due to AEs and the overall incidence of serious AEs from any cause was similar in both groups (38 vs 37\% and 52 vs 54\%, respectively). Based on these findings, the FDA and the EMA approved sorafenib for the treatment of patients with (unresectable) HCC in 2007. Exploratory subgroup analyses demonstrated that sorafenib consistently improved median OS (HR: 0.50-0.85), median TTP (HR: 0.40-0.76), except in HBV-positive patients [HR: 1.03]), and disease control rate (DCR) compared with placebo, irrespective of the etiology of underlying liver disease, baseline aminotransferases, bilirubin, AFP concentrations, ECOG PS, tumor burden (MVI and EHS), tumor stage and prior therapy $[35,36]$. However, median OS on sorafenib treatment differed between subgroups, being the highest for patients with $\mathrm{HCV}$ infection (HR: 0.50; 95\% CI: 0.32-0.77) and lowest for patients with a presence of EHS (HR: 0.85; 95\% CI: 0.64-1.15).

The use of sorafenib as the standard of care in patients with advanced HCC and maintained liver function CP A was supported by the Phase III Asia-Pacific randomized controlled trial (RCT) (Table 2) [12]. In contrast with the SHARP trial where the main etiologies of underlying liver disease were $\mathrm{HCV}$ and alcohol, this trial was performed in $\mathrm{HBV}$-endemic areas from the Asia-Pacific region. A total of 226 patients with advanced HCC (97\% CP A, 73\% HBV, 95\% BCLC C, 35.4\% MVI and $68.6 \%$ EHS) were randomly assigned to receive either sorafenib or placebo in a 2:1 fashion. The efficacy of sorafenib was overall similar to that reported in the SHARP trial, and patients receiving sorafenib had a significantly longer median OS (6.5 vs 4.2 months; HR 0.68; 95\% CI: 0.50-0.93; $\mathrm{p}=0.014)$ and median TTP (2.8 vs 1.4 months; HR: 0.57; 95\% CI: $0.42-0.79 ; \mathrm{p}=0.0005)$ compared with those receiving placebo. However, patients presented with more advanced disease (BCLC C and ECOG PS 1) and were mainly HBV infected, which might explain the lower absolute survival compared with the SHARP trial. The most frequently reported grade 3/4 drug-related AEs in patients treated with sorafenib were HFSR (10.7\%), diarrhea (6.0\%) and fatigue (3.4\%). 
Treatment discontinuation due to AEs was similar in both groups (19.5 vs 13.3\%). Exploratory subgroup analyses showed that sorafenib consistently improved median OS (HR: 0.32-0.94) and median TTP (HR: 0.31-0.75) compared with placebo, irrespective of baseline status [37]. However, median OS on sorafenib treatment differed between subgroups, again being lowest for patients with a presence of EHS (lung metastases; HR: 0.87; 95\% CI: 0.56-1.37).

\section{- Postmarketing surveillance Observational studies}

The favorable results reported in the SHARP trial regarding the efficacy and safety of sorafenib were reproduced by several observational studies in clinical practice [38-41]. GIDEON was a global, noninterventional surveillance study that aimed to evaluate sorafenib in more than 3000 patients with unresectable HCC under real-life conditions, particularly in CP B patients (who were excluded in the Phase III trials). The final analysis was presented at the American Society of Clinical Oncology 2013 meeting [38]. The incidence of AEs and drug-related AEs (mainly diarrhea, HFSR and fatigue) was similar across $\mathrm{CP}$ subgroups, although serious AEs (60.4 vs $36 \%$; drug-related 14.1 vs $8.8 \%$ ) and discontinuation due to $\mathrm{AEs}$ ( 40.1 vs $28.9 \%$ ) were more common in CP B patients. Duration of treatment was longer in CP A patients (17.6 weeks) compared with CP B patients (9.9 weeks). In the intent-to-treat population, median OS was longer in CP A patients (13.6 months; $95 \% \mathrm{CI}$ : 12.8-14.7) than in CP B patients (5.2 months; 95\% CI: 4.6-6.3), or CP C patients (2.6 months; 95\% CI: 1.5-4.0); however, TTP was similar (CP A patients 4.7 months [95\% CI: 4.3-5.2]; CP B patients 4.4 months [95\% CI: 3.5-5.5]; CP C 3.6 months [95\% CI: 2.1-6.0]) between subgroups. The authors concluded that sorafenib is safe in patients with unresectable HCC irrespective of liver function CP A/B; however, CP status may represent a strong prognostic factor regarding the OS. These data are in accordance with previous reports demonstrating a shorter treatment time and only moderate clinical benefit with respect to survival (median OS: 2.0-7.7 months) in CP B patients on sorafenib treatment [39-48]. Taken together, sorafenib treatment of CP B patients appears to be feasible with a comparable safety profile and no relevant differences regarding the TTP; however, the survival benefit remains questionable, most likely attributed to the natural course of advanced liver cirrhosis. Despite the lack of a RCT in this setting, maintained liver function seems to be a basic requirement for a meaningful sorafenib treatment in daily practice and CP B patients should only be treated with caution on an individual basis after careful evaluation. Patients with clinical signs of hepatic decompensation (ascites, encephalopathy, bilirubin $>3 \mathrm{mg} / \mathrm{dl}$ and CP score $>7$ points) and CP C patients in whom survival is not dictated by their cancer as much as by their cirrhosis are unlikely to benefit from sorafenib, and should be treated with best supportive care.

Safety and efficacy of sorafenib was validated in another large multicenter field study (SOFIA) [39]. A total of 296 patients (88\% CP A) with advanced (75\% BCLC C, 39\% MVI and 35\% EHS) or intermediate (25\% BCLC B, failed prior locoregional treatment) HCC were evaluated. Median treatment time was 3.8 months and the most common grade 3/4 AEs were fatigue (25\%), HFSR (9\%), arterial hypertension (7\%), diarrhea $(6 \%)$, weight loss $(6 \%)$ and gastrointestinal bleeding (5\%). Compared with the SHARP trial, tolerability was poorer, 161 patients (54\%) needed a dose reduction and only $44 \%$ of patients were able to continue treatment with sorafenib in the absence of tumor progression. Median OS was 10.5 months in the overall cohort, and 8.4 months in BCLC C versus 20.6 months in BCLC B patients ( $\mathrm{p}<0.0001)$. Median OS was 12.7 months for CP A patients and 7.7 months for CP B patients, respectively. Patients treated with full dose sorafenib more than $70 \%$ of the time demonstrated a shorter median OS (9.6 months) compared with patients treated with a half dose (due to AEs) for more than $70 \%$ of the time (21.6 months; $\mathrm{p}=0.0006$ ), possibly reflecting the ability of sorafenib to retain anticancer activity also at lower doses than full in those who need dose reductions due to AEs (implications for tailored therapy).

The viability of continuing sorafenib at a higher dosage in patients who experienced radiologic disease progression was investigated in a Phase II RCT [49]. In total, 101 patients with advanced HCC who experienced disease progression while on sorafenib $400 \mathrm{mg}$ twice daily were randomized to sorafenib $600 \mathrm{mg}$ twice daily or best supportive care. The study did not meet its primary end point with a PFS of 3.91 months in the sorafenib arm and 2.69 months in the best supportive care $\operatorname{arm}(\mathrm{p}=0.086)$. 
Response assessment to sorafenib treatment Several baseline characteristics could be identified as negative prognostic indicators for the OS (not predictive regarding the response) such as liver function (reflected by CP score), presence of ascites, impaired ECOG PS, elevated baseline aminotransferases, bilirubin or AFP concentrations, and particularly the presence of MVI and/or EHS [34-38,41,42,50,51]. Regarding baseline factors with a positive prognostic and predictive value, it was noted that patients with HCV-related HCC demonstrate marked benefit with respect to OS (14.0 vs 7.4 months, HR: $0.50 ; 95 \%$ CI: $0.32-0.77)$ and TTP (7.6 vs 2.8 months; HR: 0.43; 95\% CI: 0.25-0.73) compared with placebo in the SHARP trial [36] and other studies $[18,30,41]$.

Some retrospective studies have reported that the occurrence of AEs on sorafenib treatment, such as skin toxicity [52,53], diarrhea [54] or arterial hypertension [55], may represent surrogate markers of response and prognosis. Despite lacking validation, AEs should be treated consequently with symptomatic therapy or temporary dose reduction to prevent discontinuation of sorafenib treatment due to AEs in clinical practice.

Almost all patients present with radiological progression after several months of sorafenib treatment, particularly upon response assessment according to conventional RECIST. As known from the Phase II-III trials [11,12,32], sorafenib rarely produces radiological tumor regression (2-3\% ORR), pointing out limitations in using diameter measurement for the response assessment in the era of MTT, and leading to the introduction of modified RECIST (mRECIST) $[3,16,17]$. The assessment of changes in tumor viability in the arterial phase of contrast-enhanced radiological imaging (arterial enhancement) was recently evaluated in 53 patients who received sorafenib for advanced HCC [56]. ORR was 23\% with mRECIST and $2 \%$ with RECIST 1.1 , and the response according to $\mathrm{mRECIST}$ was a surrogate for the OS (18 months for responders vs 8 months for nonresponders; $\mathrm{p}=0.013$ ). In the 42 patients who achieved SD according to RECIST 1.1, OS differed depending on tumor response according to $\mathrm{mRECIST}$, with a median OS of 17,10 and 4 months for patients who achieved objective response (OR), SD and PD, respectively ( $p=0.016$ ). Based on these findings and recommendations, there is a rationale to discontinue sorafenib treatment in patients with ongoing clinical benefit in daily practice only in case of a clear radiological tumor progression according to mRECIST (increase in tumor volume without reduction of arterial enhancement or in case of [confirmed] new lesions). After discontinuation of sorafenib treatment, patients should be included in clinical studies whenever possible.

Regarding the use of (serum) biomarkers, serial AFP measurement within the first weeks of treatment may provide additional information in monitoring treatment response to sorafenib complementary to imaging-based assessment [57,58]. This approach was recently evaluated in 85 patients with advanced HCC treated with sorafenib [59]. In total, 32 patients $(37.6 \%)$ were responders ( $>20 \%$ decrease in AFP level during 8 weeks of treatment), whereas 58 patients (70.7\%) achieved radiological disease control according to RECIST. Median OS was 13.3 months among AFP responders but only 8.2 months in nonresponders ( $p=0.022)$. Median TTP was 7.9 months among responders but only 2.4 months for nonresponders $(\mathrm{p}=0.004)$. HRs for survival according to AFP response and radiological disease control were $0.59(p=0.040)$ and $1.03(p=0.913)$, respectively. However, it should be pointed out that a lack of AFP response does not necessarily indicate a lack of efficacy for sorafenib. The results of the biomarker analysis from the SHARP trial were recently reported [60]. The angiogenesis biomarkers angiopoietin 2 and VEGF were independent predictors of survival in patients with advanced HCC; however, none of them could significantly predict response to sorafenib treatment. The role of pERK as a tissue biomarker has to be further defined $[32,61]$.

\section{Sorafenib in combination studies in} advanced HCC

After its approval, sorafenib was tested in multiple Phase I-III trials in advanced HCC during the last years, either head to head or in combination with different novel agents and conventional chemotherapeutics.

\section{Sorafenib in combination with conventional chemotherapy}

Based on a Phase I study [62], the combination of doxorubicin (60 mg/m $\mathrm{m}^{2}$ every 3 weeks) plus sorafenib (400 mg twice daily) versus doxorubicin plus placebo was tested in 96 patients with advanced HCC in a Phase II RCT [63]. The 
median TTP and OS were 6.4 and 13.7 months in the doxorubicin plus sorafenib arm, and 2.8 and 6.5 months in the doxorubicin plus placebo $\operatorname{arm}(\mathrm{p}=0.02$ or $\mathrm{p}=0.006)$, respectively. A Phase III RCT (CALGB-80802) comparing sorafenib plus doxorubicin versus sorafenib alone is ongoing. In further single-arm Phase II studies, combinations of sorafenib with cytotoxic drugs such as metronomic tegafur/uracil (median PFS: 3.7 months; 95\% CI: 1.9-5.5; median OS: 7.4 months; 95\% CI: 3.4-11.4) [64], 5-FU (median TTP: 8 months; 95\% CI: 5.7-10.4; median OS: 13.7 months; 95\% CI: 9.5-17.9) [65] or capecitabine/oxaliplatin (median TTP: 5.29 months; 95\% CI: 3.81-5.88; median OS: 11.73 months; 95\% CI: 8.87-15.38) [66] have shown manageable safety profiles and moderate efficacy. Recently, the results of the GONEXT Phase II trial were presented [67]. A total of 83 patients with advanced HCC were treated either with sorafenib plus gemcitabine/oxaliplatin or with sorafenib monotherapy. However, regarding DCR, median PFS (6.2 vs 4.6 months; $\mathrm{p}=0.684$ ) and median OS (13.5 vs 13.0 months; $\mathrm{p}=0.114$ ), no significant differences were shown. Taken together, combining sorafenib with conventional cytotoxic chemotherapy appears to be feasible; however, due to the lack of a sorafenib control arm in most studies, the relative contribution of the cytotoxic drug/regimen to efficacy, if any, remains difficult to assess.

\section{Sorafenib in combination with other targeted agents}

An assumed synergistic effect by targeting both VEGFR-dependent angiogenesis and EGFRdependent tumor cell proliferation provided the rationale for evaluating the combination of sorafenib and the EGFR tyrosine kinase inhibitor erlotinib versus sorafenib plus placebo in a Phase III RCT (SEARCH) [22]. However, the trial did not meet its primary end point in improving the median OS (9.5 months for sorafenib plus erlotinib vs 8.5 months for sorafenib plus placebo; HR: 0.929; $\mathrm{p}=0.204$ ).

Enhanced efficacy may also be achieved through dual inhibition of MAPK and PI3K/Akt/ mTOR signaling pathway. In a Phase I study, the MTD of the mTOR inhibitor everolimus in combination with sorafenib in 30 patients with advanced HCC was evaluated using escalating doses of everolimus starting at $2.5 \mathrm{mg}$ daily plus sorafenib $400 \mathrm{mg}$ twice daily [68]. Dose escalation above everolimus $5 \mathrm{mg}$ daily was not achieved. All patients experienced one or more AE, most commonly diarrhea (66.7\%), HFSR (66.7\%) and thrombocytopenia $(50.0 \%)$. Best overall response was SD (62.5 and $42.9 \%$ in the $2.5 \mathrm{mg}$ and $5.0 \mathrm{mg}$ cohorts, respectively). Median TTP and OS in the $2.5 \mathrm{mg}$ cohort were 4.5 and 7.4 months, and 1.8 and 11.7 months in the $5.0 \mathrm{mg}$ cohort, respectively. The authors concluded that the MTD of everolimus in combination with sorafenib was $2.5 \mathrm{mg}$ daily and the inability to achieve what is deemed a biologically effective everolimus concentration precluded progression to Phase II with this combination. Explanations for the low MTD may be the hepatic impairment (compared with studies in patients with advanced RCC) and the higher percentage of Asian patients with HBV infection. In line with these assumptions, maintained liver function might explain the promising results from the combination of sorafenib and mTOR inhibitors (sirolimus and everolimus) from retrospective studies in patients with HCC recurrence after liver transplantation [69-71]. These studies demonstrated an acceptable safety profile and an assumed survival benefit in these patients. No deterioration of liver graft function occurred; however, permanent sorafenib dose reduction to $200 \mathrm{mg}$ twice daily was necessary in many patients. Less information is available regarding the use of sorafenib in the pretransplant setting [72]. A Phase II RCT (sorafenib vs placebo) in the adjuvant setting after liver transplantation for HCC in high-risk patients is ongoing [73].

With regard to epigenetic alterations, the histone deacetylase inhibitor resminostat was tested in a Phase II study (SHELTER) [74]. Patients with advanced HCC after progression on prior sorafenib treatment received $600 \mathrm{mg}$ resminostat either alone or in combination with sorafenib $400 \mathrm{mg}$ twice daily. The primary end point (20\% PFS rate at 12 weeks) was reached in the combination arm (70\%) with a median PFS of 4.7 months and a median OS of 8.4 months, respectively. Most frequently observed drug-related AEs were nausea (grade 3/4 15\%), vomiting, diarrhea and fatigue.

\section{Sorafenib in earlier stages of the disease}

Sorafenib is under investigation in earlier stages of the disease in a Phase III RCT (STORM) in the adjuvant setting after potentially curative resection or local ablation, with the aim to decrease the high rate of recurrence (up to $70 \%$ within 5 years) after these therapies. 
In addition, there is a strong rationale to combine TACE with antiangiogenic agents such as sorafenib in the intermediate stage of the disease (BCLC B). Most patients present with disease relapse on or experience disease progression after TACE with a median OS of around 20 months $[3,6]$. The peripheral part of the tumor has a tendency to survive TACE, and hypoxia in the surviving margin may upregulate growth and angiogenic factors (e.g., HIF-1 $\alpha$ or VEGF) leading to rebound neovascularization [75]. Hypothetically, sorafenib might curtail this post-TACE rise in VEGF-mediated signaling, leading to improved outcome [76]. However, many studies in this setting during recent years have been small, openlabel, uncontrolled single-arm, Phase I-II trials with inconclusive results. They have used different end points (TTP, OS and safety), different response assessments (RECIST and mRECIST), heterogeneous TACE procedures (conventional TACE [77-82] versus drug-eluting beads [DEB]TACE [83-85]) and schedules (predefined vs ondemand), heterogeneous sorafenib schedules (sequential [77,78] vs interrupted [79,80] vs continuous [81-85]), and included heterogeneous patients with respect to stage (BCLC B vs BCLC C $[86,87]$ ), and liver function (CP A vs $\mathrm{CP}$ B). Most recently, a Phase II RCT (SPACE) combining DEB-TACE with sorafenib compared with DEB-TACE with placebo met its primary end point of modest improvement in TTP (169 vs 166 days) with a HR of 0.797 (95\% CI: $0.588-1.08 ; \mathrm{p}=0.072$ ) and a one-sided $\alpha$ of 0.15 [85]. Taken together, results regarding the combination of TACE and sorafenib are inconclusive; particularly randomized controlled data are lacking. However, the combination of TACE with sorafenib appears to be safe and feasible, and safety profiles might be similar to those of sorafenib and TACE alone in most cases without any relevant additive toxicity. Preliminary efficacy was shown, particularly in patients treated with sorafenib for longer periods of time; however, results of ongoing Phase III RCTs (TACE-2 and ECOG-E1208) are needed to clarify the clinical implication of this combined treatment approach in intermediate HCC. This is particularly true for the situation in advanced $\mathrm{HCC}[86,87]$. These assumptions were recently reinforced by a systematic review of this topic [88].

\section{- Safety \& tolerability}

Sorafenib exhibits a broad range of AEs including gastrointestinal (diarrhea, nausea and gastrointestinal perforation), dermatological (HFSR, dry skin, rash, pruritus and hair loss), constitutional (fatigue and weight loss), cardiovascular (arterial hypertension and myocardial infarction) and hemorrhagic [11,12,29,32,89]; however, the most common AEs of all grades in clinical practice are diarrhea, HFSR and fatigue. Although a slightly higher rate of AEs were reported in observational studies [38,39] when compared with the Phase III trials, most common AEs are usually grade $1 / 2$ according to Common Terminology Criteria of Adverse Events and well manageable in clinical practice. However, relevant toxicities can occur and negatively affect both patient quality of life and the efficacy of sorafenib treatment.

The recommended dose of $400 \mathrm{mg}$ sorafenib twice daily may be administered in most patients; however, it is also possible to start at a reduced dose (200 mg twice daily) and titrate to the target dose after the patient has been proven to tolerate the drug [90,91].

For the prevention of most common and predictable AEs (diarrhea and skin reactions), patients should avoid fatty/spicy meals and ther$\mathrm{mal} /$ mechanical skin stimuli, and should receive prescriptions for loperamide and topical ureacontaining (5-10\%) ointments. In addition, careful patient education is crucial to improve adherence to treatment. Clinical visits (including stool diary, blood pressure monitoring and laboratory follow-up, including liver function tests) should be performed every 2 to 4 weeks on an outpatient basis for the early diagnosis of relevant toxicities.

Once established, most grade 1/2 AEs can be relieved by symptomatic treatment. Diarrhea (small intestinal bacterial overgrowth is suggested as a possible pathophysiological mechanism [92]) should be treated with loperamide (adequate dose) and sufficient oral rehydration. HFSR (generally appearing during the first weeks of treatment) and rash can be treated with topical urea-containing ointments or corticosteroids [93]. Thermal (hot water) and mechanical (pressure to sensitive areas) skin stimuli should be avoided. Arterial hypertension is amenable in most cases to standard antihypertensive medication. Management of fatigue, which can be related to cancer, underlying liver cirrhosis and (less likely) sorafenib treatment, is challenging. Coexisting morbidities such as hypothyroidism, anemia, malnutrition and depression have to be carefully 
ruled out in these patients. Patients should, if possible, be encouraged to partake in physical activity and sufficient caloric intake.

Grade 3/4 AEs may require temporary dose reduction $(200 \mathrm{mg}$ twice daily or $400 \mathrm{mg}$ every other day) or treatment interruption, or in severe or persistent cases, permanent discontinuation. Treatment should be interrupted until toxicity resolves to grade $0 / 1$. In persistent cases, patients may be treated with a permanent dose reduction instead of discontinuation because certain AEs might represent surrogate parameters for response and prognosis [52-55] and, as suggested from the SOFIA trial, lower doses of sorafenib might retain anticancer activity in those who need dose reductions due to AEs [39]. Hemorrhagic events are infrequent in patients receiving sorafenib and are mainly due to underlying portal hypertension and impaired coagulation. Complications of portal hypertension, such as esophageal varices, should therefore be evaluated before initiation of sorafenib treatment. If bleeding causes medical intervention, permanent discontinuation of sorafenib should be considered.

\section{- Regulatory affairs}

The FDA indicate that sorafenib is used for the treatment of patients with unresectable HCC and advanced RCC. The EMA indicate that sorafenib be used for the treatment of HCC. Sorafenib is indicated for the treatment of patients with advanced RCC who have failed prior IFN- $\alpha$ or IL-2 based therapy or are considered unsuitable for such therapy. Sorafenib is not reimbursed in patients with HCC by NICE and the Scottish Medicines Consortium in the UK.

Regarding the cost-effectiveness of sorafenib, two relevant studies were published during recent years. Based on the results of the SHARP trial, the economic evaluation demonstrated that sorafenib is cost effective compared with best supportive care in patients with advanced HCC, with a cost-effectiveness ratio within the established threshold that the US society is willing to pay, and significantly lower than alternative thresholds suggested in recent years in oncology [94]. By contrast, full-dose sorafenib was shown not to be cost effective in intermediate and advanced HCC patients in a recent subanalysis of the large multicenter field SOFIA study. Instead, dose-adjusted sorafenib was cost effective in patients with advanced HCC over a wide range of model assumptions, but not in those with intermediate HCC who were not eligible to or failed locoregional therapy [95]. As in other agents [96], the cost-effectiveness of sorafenib may be worse when the analysis is based on data from actual clinical practice (SOFIA) rather than RCTs (SHARP). This relevant topic needs further validation.

\section{Conclusion}

The multikinase inhibitor sorafenib was the first systemic agent to demonstrate a significant improvement in the OS for patients with advanced HCC. Survival benefits reported in two Phase III RCTs were reproduced by several observational studies in clinical practice establishing sorafenib as the standard of care in CP A patients with advanced HCC or in those ineligible for or after failure of locoregional therapies in the intermediate stage of the disease. However, several baseline characteristics could be identified as negative prognostic indicators for OS, first and foremost impaired liver function. Treatment of CP B patients might be feasible in clinical practice; however, the survival benefit remains questionable. AEs (mainly diarrhea, HFSR and fatigue) are manageable in most cases in daily practice with careful patient education, preventive measures and early diagnosis. Most grade $1 / 2$ AEs can be relieved by symptomatic treatment; however, grade 3/4 AEs may require temporary dose reduction or treatment interruption, or in severe or persistent cases, permanent discontinuation. If patients achieve ongoing clinical benefit from sorafenib, treatment should be discontinued only in case of clear tumor progression according to mRECIST, which should be considered for radiological assessment of treatment response in the era of MTT. After discontinuation of sorafenib treatment, patients should be in included in clinical studies, whenever possible.

The advent of sorafenib has broadened the horizon for patients with HCC. However, survival benefit of sorafenib treatment in the advanced stage of the disease remains modest in clinical practice and its role in the earlier stages of disease (in the adjuvant setting or in combination with TACE) has to be defined. Treatment decisions based on a multidisciplinary background, optimal management of AEs, and careful evaluation of radiological treatment response are crucial to optimize the outcome of patients treated with sorafenib in clinical practice. To 
further improve the benefits of sorafenib, the identification of clinical and molecular markers predicting response and prognosis on sorafenib treatment are urgently needed.

No standard treatment option currently exists outside of clinical trials for patients with acquired resistance or intolerance to sorafenib. The failure of sunitinib [18], linifanib [19], brivanib $[20,21]$ and erlotinib [22] in recent Phase III trials, despite signs of activity (mirrored by improvements in TTP, ORR and DCR) in the firstand second-line setting, demonstrates that carefully designed Phase II RCTs in novel targeted agents are needed to detect early evidence of safety and efficacy that will translate into survival benefit in the Phase III setting. Knowledge of the current landscape of MTT in advanced HCC underscores the need to develop more active agents with novel mechanisms of action and more favorable safety profiles. Molecular alterations in hepatocarcinogenesis may differ depending on etiology, activity and duration of the underlying liver injury, hereby influencing response to therapy. Therefore, every effort should be undertaken to unravel the key signaling cascades, identify molecular tissue biomarkers that are both prognostic and predictive, and classify HCC patients into subgroups as demonstrated in the Phase II trial of the c-MET tyrosine kinase inhibitor tivantinib [97]. This biomarker-driven approach bears the potential to individualize anticancer therapy (personalized medicine), in the end allowing treatment of those benefiting most and excluding those who do not. Routine biopsy prior to enrollment in clinical trials on MTT is essential in the future to avoid the high risk of failure in unselected populations and to finally improve the survival of patients with advanced HCC beyond the 1-year mark [15]. Until then, sorafenib remains the standard of care in $\mathrm{CP}$ A patients with advanced HCC or in those ineligible for or after failure of locoregional therapies in the intermediate stage of the disease.

Financial \& competing interests disclosure MA Wörns and PR Galle have received consulting and lecture fees from Bayer HealthCare (Leverkusen, Germany). The authors have no other relevant affiliations or financial involvement with any organization or entity with a financial interest in or financial conflict with the subject matter or materials discussed in the manuscript apart from those disclosed.

No writing assistance was utilized in the production of this manuscript.

\section{References}

Papers of special note have been highlighted as:

- of interest

-. of considerable interest

1 Jemal A, Bray F, Center MM, Ferlay J, Ward E, Forman D. Global cancer statistics. $C A$ Cancer J. Clin. 61(2), 69-90 (2011).

2 Alazawi W, Cunningham M, Dearden J, Foster GR. Systematic review: outcome of compensated cirrhosis due to chronic hepatitis C infection. Aliment. Pharmacol. Ther. 32(3), 344-355 (2010)

3 European Association For The Study Of The Liver; European Organisation For Research And Treatment Of Cancer. EASL-EORTC clinical practice guidelines: management of hepatocellular carcinoma. J. Hepatol. 56(4), 908-943 (2012).

- Current European Association for the Study of the Liver-European Organisation for Research and Treatment of Cancer guidelines regarding the management of hepatocellular carcinoma (HCC).

4 Forner A, Llovet JM, Bruix J. Hepatocellular carcinoma. Lancet 379(9822), 1245-1255 (2012).
5 Llovet JM, Bru C, Bruix J. Prognosis of hepatocellular carcinoma: the BCLC staging classification. Semin. Liver Dis. 19(3), 329338 (1999).

6 Llovet JM, Bruix J. Systematic review of randomized trials for unresectable hepatocellular carcinoma: chemoembolization improves survival. Hepatology 37(2), 429-442 (2003).

7 Bruix J, Sherman M. Management of hepatocellular carcinoma: an update. Hepatology 53(3), 1020-1022 (2011).

- Current American Association for the Study of Liver Diseases guidelines regarding the management of HCC.

8 Worns MA, Weinmann A, Schuchmann M, Galle PR. Systemic therapies in hepatocellular carcinoma. Dig. Dis. 27(2), 175-188 (2009).

9 Worns MA, Galle PR. Novel inhibitors in development for hepatocellular carcinoma. Expert Opin. Investig. Drugs 19(5), 615-629 (2010).

10 Villanueva A, Newell P, Chiang DY, Friedman SL, Llovet JM. Genomics and signaling pathways in hepatocellular carcinoma. Semin. Liver Dis. 27(1), 55-76 (2007).
11 Llovet JM, Ricci S, Mazzaferro V et al. Sorafenib in advanced hepatocellular carcinoma. N. Engl. J. Med. 359(4), 378-390 (2008).

-1. Pivotal Phase III trial establishing sorafenib as the standard of care in advanced HCC.

12 Cheng AL, Kang YK, Chen Z et al. Efficacy and safety of sorafenib in patients in the AsiaPacific region with advanced hepatocellular carcinoma. a Phase III randomised, doubleblind, placebo-controlled trial. Lancet Oncol. 10(1), 25-34 (2009).

- Important Phase III trial further establishing sorafenib as the standard of care in advanced HCC.

13 Wilhelm SM, Carter C, Tang L et al. BAY 43-9006 exhibits broad spectrum oral antitumor activity and targets the RAF/MEK/ ERK pathway and receptor tyrosine kinases involved in tumor progression and angiogenesis. Cancer Res. 64(19), 7099-7109 (2004).

14 EMA. Nexavar ${ }^{\circledR}$. European public assessment report.

www.ema.europa.eu/ema/index. jsp?curl=pages/medicines/human/ medicines/000690/human_med_000929. jsp\&mid=WC0b01ac058001d124 
15 Worns MA. Systemic therapy and synergies by combination. Dig. Dis. 31(1), 104-111 (2013).

16 Llovet JM, Di Bisceglie AM, Bruix J et al. Design and endpoints of clinical trials in hepatocellular carcinoma. J. Natl Cancer Inst. 100(10), 698-711 (2008).

-1 Guidelines for clinical trial design in HCC from an expert panel.

17 Lencioni R, Llovet JM. Modified RECIST (mRECIST) assessment for hepatocellular carcinoma. Semin. Liver Dis. 30(1), 52-60 (2010).

18 Cheng AL, Kang Y, Lin D et al. Phase III trial of sunitinib $(\mathrm{Su})$ versus sorafenib (So) in advanced hepatocellular carcinoma (HCC). J. Clin. Oncol. 29(Suppl.), Abstract 4000 (2011).

- Phase III trial of sunitinib in the first-line setting in advanced HCC.

19 Cainap C, Qin S, Huang WT et al. Phase III trial of linifanib versus sorafenib in patients with advanced hepatocellular carcinoma (HCC). J. Clin. Oncol. 30(Suppl. 34), Abstract 249 (2013).

20 Johnson PJ, Qin S, Park JW et al. Brivanib versus sorafenib as first-line therapy in patients with unresectable, advanced hepatocellular carcinoma. results from the randomized Phase III BRISK-FL study. J. Clin. Oncol. 31(28), 3517-3524 (2013).

- Phase III trial of brivanib in the first-line setting in advanced HCC.

21 Llovet JM, Decaens T, Raoul JL et al. Brivanib in patients with advanced hepatocellular carcinoma who were intolerant to sorafenib or for whom sorafenib failed. results from the randomized Phase III BRISKPS study. J. Clin. Oncol. 31(28), 3509-3516 (2013).

- Phase III trial of brivanib in the second-line setting in advanced HCC.

22 Zhu AX, Rosmorduc O, Evans J et al. SEARCH. A Phase III, randomized, doubleblind, placebo-controlled trial of sorafenib plus erlotinib in patients with advanced hepatocellular carcinoma (HCC). Eur. J. Cancer 48(Suppl.), LBA2 (2012).

- Phase III trial of sorafenib plus erlotinib in the first-line setting in advanced HCC.

23 Wilhelm SM, Adnane L, Newell P, Villanueva A, Llovet JM, Lynch M. Preclinical overview of sorafenib, a multikinase inhibitor that targets both Raf and VEGF and PDGF receptor tyrosine kinase signaling. Mol. Cancer Ther. 7(10), 3129-3140 (2008).

24 Carlomagno F, Anaganti S, Guida T et al. BAY 43-9006 inhibition of oncogenic RET mutants. J. Natl Cancer Inst. 98(5), 326-334 (2006).

25 Yu C, Bruzek LM, Meng XW et al. The role of Mcl-1 downregulation in the proapoptotic activity of the multikinase inhibitor BAY 43 9006. Oncogene 24(46), 6861-6869 (2005).

26 Liu L, Cao Y, Chen C et al. Sorafenib blocks the RAF/MEK/ERK pathway, inhibits tumor angiogenesis, and induces tumor cell apoptosis in hepatocellular carcinoma model PLC/ PRF/5. Cancer Res. 66(24), 11851-11858 (2006).

27 Rahmani M, Davis EM, Bauer C, Dent P, Grant $S$. Apoptosis induced by the kinase inhibitor BAY 43-9006 in human leukemia cells involves down-regulation of Mcl-1 through inhibition of translation. J. Biol. Chem. 280(42), 35217-35227 (2005).

28 Sprinzl MF, Reisinger F, Puschnik A et al. Sorafenib perpetuates cellular anticancer effector functions by modulating the crosstalk between macrophages and natural killer cells. Hepatology 57(6), 2358-2368 (2013).

29 Strumberg D, Clark JW, Awada A et al. Safety, pharmacokinetics, and preliminary antitumor activity of sorafenib. a review of four Phase I trials in patients with advanced refractory solid tumors. Oncologist 12(4), 426-437 (2007).

- Comprehensive overview regarding Phase I studies of sorafenib in solid tumors.

30 Furuse J, Ishii H, Nakachi K, Suzuki E, Shimizu S, Nakajima K. Phase I study of sorafenib in Japanese patients with hepatocellular carcinoma. Cancer Sci. 99(1), 159-165 (2008).

31 Miller AA, Murry DJ, Owzar K et al. Phase I and pharmacokinetic study of sorafenib in patients with hepatic or renal dysfunction. CALGB 60301. J. Clin. Oncol. 27(11), 1800-1805 (2009).

32 Abou-Alfa GK, Schwartz L, Ricci S et al. Phase II study of sorafenib in patients with advanced hepatocellular carcinoma. J. Clin. Oncol. 24(26), 4293-4300 (2006).

-1" Phase II study of sorafenib in advanced HCC.

33 Abou-Alfa GK, Amadori D, Santoro A et al. Safety and efficacy of sorafenib in patients with hepatocellular carcinoma (HCC) and Child-Pugh A versus B cirrhosis. Gastrointest. Cancer Res. 4(2), 40-44 (2011).

-1. Phase II study of sorafenib in advanced HCC.

34 Yau T, Chan P, Ng KK et al. Phase 2 openlabel study of single-agent sorafenib in treating advanced hepatocellular carcinoma in a hepatitis B-endemic Asian population. presence of lung metastasis predicts poor response. Cancer 115(2), 428-436 (2009).

35 Raoul JL, Bruix J, Greten TF et al. Relationship between baseline hepatic status and outcome, and effect of sorafenib on liver function. SHARP trial subanalyses. J. Hepatol. 56(5), 1080-1088 (2012).

- Subgroup analyses from the Phase III trial of sorafenib in advanced HCC.

36 Bruix J, Raoul JL, Sherman M et al. Efficacy and safety of sorafenib in patients with advanced hepatocellular carcinoma. subanalyses of a Phase III trial. J. Hepatol. 57(4), 821-829 (2012).

- Subgroup analyses from the Phase III trial of sorafenib in advanced HCC.

37 Cheng AL, Guan Z, Chen Z et al. Efficacy and safety of sorafenib in patients with advanced hepatocellular carcinoma according to baseline status. Subset analyses of the Phase III sorafenib Asia-Pacific trial. Eur. J. Cancer 48(10), 1452-1465 (2012).

- Subgroup analyses from the Phase III trial of sorafenib in advanced HCC.

38 Marrero JA, Lencioni R, Ye SL et al. Final analysis of GIDEON (Global Investigation of Therapeutic Decisions in Hepatocellular Carcinoma [HCC] and of Its Treatment with Sorafenib [Sor]) in $>3000$ Sor-treated patients (pts): Clinical findings in pts with liver dysfunction. J. Clin. Oncol. 31(Suppl.), Abstract 4126 (2013).

- Large observational study regarding sorafenib in clinical practice.

39 Iavarone M, Cabibbo G, Piscaglia F et al. Field-practice study of sorafenib therapy for hepatocellular carcinoma. a prospective multicenter study in Italy. Hepatology 54(6), 2055-2063 (2011).

- Large observational study regarding sorafenib in clinical practice.

40 Pressiani T, Boni C, Rimassa L et al. Sorafenib in patients with Child-Pugh class A and $\mathrm{B}$ advanced hepatocellular carcinoma. a prospective feasibility analysis. Ann. Oncol. 24(2), 406-411 (2013).

41 Worns MA, Koch S, Niederle IM et al. The impact of patient and tumour baseline characteristics on the overall survival of patients with advanced hepatocellular carcinoma treated with sorafenib. Dig. Liver Dis. 45(5), 408-413 (2013).

42 Pinter M, Sieghart W, Graziadei I et al. Sorafenib in unresectable hepatocellular carcinoma from mild to advanced stage liver cirrhosis. Oncologist 14(1), 70-76 (2009). 
43 Kim JE, Ryoo BY, Ryu MH et al. Sorafenib for hepatocellular carcinoma according to ChildPugh class of liver function. Cancer Chemother. Pharmacol. 68(5), 1285-1290 (2011).

44 Hollebecque A, Cattan S, Romano O et al. Safety and efficacy of sorafenib in hepatocellular carcinoma. the impact of the Child-Pugh score. Aliment. Pharmacol. Ther. 34(10), 1193-1201 (2011).

45 Ozenne V, Paradis V, Pernot S et al. Tolerance and outcome of patients with unresectable hepatocellular carcinoma treated with sorafenib. Eur. J. Gastroenterol. Hepatol. 22(9), 1106-1110 (2010).

46 Zugazagoitia J, Manzano A, Sastre J, Ladero JM, Puente J, Diaz-Rubio E. Sorafenib for non-selected patient population with advanced hepatocellular carcinoma: efficacy and safety data according to liver function. Clin. Transl Oncol. 15(2), 146-153 (2013).

47 Kostner AH, Sorensen M, Olesen RK, Gronbaek H, Lassen U, Ladekarl M. Sorafenib in advanced hepatocellular carcinoma: a nationwide retrospective study of efficacy and tolerability. ScientificWorldJournal 2013, 931972 (2013)

48 Chiu J, Tang YF, Yao TJ et al. The use of single-agent sorafenib in the treatment of advanced hepatocellular carcinoma patients with underlying Child-Pugh B liver cirrhosis. a retrospective analysis of efficacy, safety, and survival benefits. Cancer 118(21), 5293-5301 (2012).

49 Rimassa L, Pressiani T, Boni C et al. A Phase II randomized dose escalation trial of sorafenib in patients with advanced hepatocellular carcinoma. Oncologist 18(4), 379-380 (2013).

50 Song T, Zhang W, Wu Q, Kong D, Ma W. A single center experience of sorafenib in advanced hepatocellular carcinoma patients: evaluation of prognostic factors. Eur. J. Gastroenterol. Hepatol. 23(12), 1233-1238 (2011)

51 Cho JY, Paik YH, Lim HY et al. Clinical parameters predictive of outcomes in sorafenib-treated patients with advanced hepatocellular carcinoma. Liver Int. 33(6), 950-957 (2013).

52 Otsuka T, Eguchi Y, Kawazoe S et al. Skin toxicities and survival in advanced hepatocellular carcinoma patients treated with sorafenib. Hepatol. Res. 42(9), 879-886 (2012).

53 Vincenzi B, Santini D, Russo A et al. Early skin toxicity as a predictive factor for tumor control in hepatocellular carcinoma patients treated with sorafenib. Oncologist 15(1), 85-92 (2010).
54 Bettinger D, Schultheiss M, Knuppel E, Thimme R, Blum HE, Spangenberg HC. Diarrhea predicts a positive response to sorafenib in patients with advanced hepatocellular carcinoma. Hepatology 56(2), 789-790 (2012).

55 Estfan B, Byrne M, Kim R. Sorafenib in advanced hepatocellular carcinoma: hypertension as a potential surrogate marker for efficacy. Am. J. Clin. Oncol. 36(4), 319324 (2013).

56 Edeline J, Boucher E, Rolland Y et al. Comparison of tumor response by Response Evaluation Criteria in Solid Tumors (RECIST) and modified RECIST in patients treated with sorafenib for hepatocellular carcinoma. Cancer 118(1), 147-156 (2012).

- Role of modified Response Evaluation Criteria in Solid Tumors for the assessment of response to sorafenib treatment.

57 Yau T, Yao TJ, Chan P et al. The significance of early alpha-fetoprotein level changes in predicting clinical and survival benefits in advanced hepatocellular carcinoma patients receiving sorafenib. Oncologist 16(9), 1270-1279 (2011).

58 Shao YY, Lin ZZ, Hsu C, Shen YC, Hsu $\mathrm{CH}$, Cheng AL. Early alpha-fetoprotein response predicts treatment efficacy of antiangiogenic systemic therapy in patients with advanced hepatocellular carcinoma. Cancer 116(19), 4590-4596 (2010).

59 Personeni N, Bozzarelli S, Pressiani T et al. Usefulness of alpha-fetoprotein response in patients treated with sorafenib for advanced hepatocellular carcinoma. J. Hepatol. 57(1), 101-107 (2012)

- Role of AFP for the assessment of response to sorafenib treatment.

60 Llovet JM, Pena CE, Lathia CD, Shan M, Meinhardt G, Bruix J. Plasma biomarkers as predictors of outcome in patients with advanced hepatocellular carcinoma. Clin. Cancer Res. 18(8), 2290-2300 (2012).

61 Personeni N, Rimassa L, Pressiani T et al. Molecular determinants of outcome in sorafenib-treated patients with hepatocellular carcinoma. J. Cancer Res. Clin. Oncol. 139(7), 1179-1187 (2013).

62 Richly H, Schultheis B, Adamietz IA et al. Combination of sorafenib and doxorubicin in patients with advanced hepatocellular carcinoma. results from a Phase I extension trial. Eur. J. Cancer 45(4), 579-587 (2009).

63 Abou-Alfa GK, Johnson P, Knox JJ et al. Doxorubicin plus sorafenib vs doxorubicin alone in patients with advanced hepatocellular carcinoma. a randomized trial. JAMA 304(19), 2154-2160 (2010).

64 Hsu CH, Shen YC, Lin ZZ et al. Phase II study of combining sorafenib with metronomic tegafur/uracil for advanced hepatocellular carcinoma. J. Hepatol. 53(1), 126-131 (2010).

65 Petrini I, Lencioni M, Ricasoli M et al. Phase II trial of sorafenib in combination with 5 -fluorouracil infusion in advanced hepatocellular carcinoma. Cancer Chemother. Pharmacol. 69(3), 773-780 (2012).

66 Yau TC, Cheung FY, Lee F et al. A multicenter Phase II study of sorafenib, capecitabine, and oxaliplatin (SECOX) in patients with advanced hepatocellular carcinoma. Final results of Hong-Kong-Singapore Hepatocellular Carcinoma Research Collaborative Group study. J. Clin. Oncol. 31(Suppl.), Abstract 4117 (2013).

67 Assenat E, Boige V, Pageaux GP et al. Sorafenib (S) alone versus $S$ combined with gemcitabine and oxaliplatin (GEMOX) in first-line treatment of advanced hepatocellular carcinoma (HCC). Final analysis of the randomized Phase II GONEXT trial (UNICANCER/FFCD PRODIGE 10 trial). J. Clin. Oncol. 31(Suppl.), Abstract 4028 (2013).

68 Finn RS, Poon RT, Yau T et al. Phase I study investigating everolimus combined with sorafenib in patients with advanced hepatocellular carcinoma. J. Hepatol. 59(6), 1271-1277 (2013).

69 Gomez-Martin C, Bustamante J, Castroagudin JF et al. Efficacy and safety of sorafenib in combination with mammalian target of rapamycin inhibitors for recurrent hepatocellular carcinoma after liver transplantation. Liver Transpl. 18(1), 45-52 (2012).

- Sorafenib treatment for HCC recurrence after liver transplantation.

70 Weinmann A, Niederle IM, Koch S et al. Sorafenib for recurrence of hepatocellular carcinoma after liver transplantation. Dig. Liver Dis. 44(5), 432-437 (2012).

71 Sposito C, Mariani L, Germini A et al. Comparative efficacy of sorafenib versus best supportive care in recurrent hepatocellular carcinoma after liver transplantation. A case-control study. J. Hepatol. 59(1), 59-66 (2013).

- Sorafenib treatment for HCC recurrence after liver transplantation.

72 Truesdale AE, Caldwell SH, Shah NL et al. Sorafenib therapy for hepatocellular carcinoma prior to liver transplant is associated with increased complications after 
transplant. Transpl. Int. 24(10), 991-998 (2011).

73 ClinicalTrials.gov. www.clinicaltrials.gov

74 Bitzer M, Giannini EG, Horger $M$ et al. Efficacy, tolerability and pharmakokinetics of the oral histone deacetylase inhibitor resminostat in patients with advanced hepatocellular carcinoma. clinical data from the Phase 2 Shelter study. Presented at: Sixth ILCA Annual Conference, Berlin, Germany, 14-16 September 2012 (Abstract 030).

75 Sergio A, Cristofori C, Cardin R et al. Transcatheter arterial chemoembolization (TACE) in hepatocellular carcinoma (HCC). the role of angiogenesis and invasiveness. $\mathrm{Am}$. J. Gastroenterol. 103(4), 914-921 (2008).

76 Strebel BM, Dufour JF. Combined approach to hepatocellular carcinoma: a new treatment concept for nonresectable disease. Expert Rev. Anticancer Ther. 8(11), 1743-1749 (2008).

-1 Perspectives in combined treatment approaches with transarterial chemoembolization and sorafenib.

77 Sansonno D, Lauletta G, Russi S, Conteduca V, Sansonno L, Dammacco F. Transarterial chemoembolization plus sorafenib: a sequential therapeutic scheme for $\mathrm{HCV}$ related intermediate-stage hepatocellular carcinoma: a randomized clinical trial. Oncologist 17(3), 359-366 (2012).

78 Kudo M, Imanaka K, Chida N et al. Phase III study of sorafenib after transarterial chemoembolisation in Japanese and Korean patients with unresectable hepatocellular carcinoma. Eur. J. Cancer 47(14), 2117-2127 (2011).

79 Park JW, Koh YH, Kim HB et al. Phase II study of concurrent transarterial chemoembolization and sorafenib in patients with unresectable hepatocellular carcinoma. J. Hepatol. 56(6), 1336-1342 (2012).

80 Chung YH, Han G, Yoon JH et al. Interim analysis of START. Study in Asia of the combination of TACE (transcatheter arterial chemoembolization) with sorafenib in patients with hepatocellular carcinoma trial. Int. J. Cancer 132(10), 2448-2458 (2013).

81 Dufour JF, Hoppe H, Heim MH et al. Continuous administration of sorafenib in combination with transarterial chemoembolization in patients with hepatocellular carcinoma. results of a Phase I study. Oncologist 15(11), 1198-1204 (2010).

82 Sieghart W, Pinter M, Reisegger $\mathrm{M}$ et al. Conventional transarterial chemoembolisation in combination with sorafenib for patients with hepatocellular carcinoma. a pilot study. Eur. Radiol. 22(6), 1214-1223 (2012).

83 Pawlik TM, Reyes DK, Cosgrove D, Kamel IR, Bhagat N, Geschwind JF. Phase II trial of sorafenib combined with concurrent transarterial chemoembolization with drugeluting beads for hepatocellular carcinoma. J. Clin. Oncol. 29(30), 3960-3967 (2011).

84 Cabrera R, Pannu DS, Caridi J et al. The combination of sorafenib with transarterial chemoembolisation for hepatocellular carcinoma. Aliment. Pharmacol. Ther. 34(2), 205-213 (2011).

85 Lencioni R, Llovet JM, Han G et al. Sorafenib or placebo in combination with transarterial chemoembolization (TACE) with doxorubicin-eluting beads (DEBDOX) for intermediate-stage hepatocellular carcinoma (HCC). Phase II, randomized, double-blind SPACE trial. J. Clin. Oncol. 30 (Suppl.), LBA154 (2012).

-1. Phase II trial regarding the combined treatment approach with drug-eluting beads transarterial chemoembolization and sorafenib in intermediate HCC.

86 Zhao Y, Wang WJ, Guan S et al. Sorafenib combined with transarterial chemoembolization for the treatment of advanced hepatocellular carcinoma. a largescale multicenter study of 222 patients. Ann. Oncol. 24(7), 1786-1792 (2013).

$87 \mathrm{Qu} \mathrm{XD}$, Chen CS, Wang JH et al. The efficacy of TACE combined sorafenib in advanced stages hepatocellullar carcinoma. BMC Cancer 12, 263 (2012).

88 Abdel-Rahman O, Elsayed ZA.

Combination trans arterial chemoembolization (TACE) plus sorafenib for the management of unresectable hepatocellular carcinoma: a systematic review of the literature. Dig. Dis. Sci. 58(12), 3389-3396 (2013).

89 Edmonds K, Hull D, Spencer-Shaw A et al. Strategies for assessing and managing the adverse events of sorafenib and other targeted therapies in the treatment of renal cell and hepatocellular carcinoma. recommendations from a European nursing task group. Eur. J. Oncol. Nurs. 16(2), 172-184 (2012).

-. Management of adverse events on sorafenib treatment.

90 Sacco R, Bargellini I, Ginanni B et al. Longterm results of sorafenib in advanced-stage hepatocellular carcinoma: what can we learn from routine clinical practice? Expert Rev. Anticancer Ther. 12(7), 869-875 (2012).

91 Kim JE, Ryoo BY, Ryu MH et al. Sorafenib dose escalation in the treatment of advanced hepatocellular carcinoma. Oncology 82(2), 119-125 (2012).

92 Novi M, Lauritano C, Rinninella E et al. Small intestinal bacterial overgrowth as a predicitve facotr for development of diarrhea in HCC patients treated with sorafenib. Hepatology 50(Suppl.), Abstract 1760 (2009).

93 Lacouture ME, Wu S, Robert C et al. Evolving strategies for the management of hand-foot skin reaction associated with the multitargeted kinase inhibitors sorafenib and sunitinib. Oncologist 13(9), 1001-1011 (2008).

94 Carr BI, Carroll S, Muszbek N, Gondek K. Economic evaluation of sorafenib in unresectable hepatocellular carcinoma. J. Gastroenterol. Hepatol. 25(11), 1739-1746 (2010).

95 Camma C, Cabibbo G, Petta S et al. Costeffectiveness of sorafenib treatment in field practice for patients with hepatocellular carcinoma. Hepatology 57(3), 1046-1054 (2013).

96 Van Staa TP, Leufkens HG, Zhang B, Smeeth L. A comparison of cost effectiveness using data from randomized trials or actual clinical practice: selective cox-2 inhibitors as an example. PLoS Med. 6(12), e1000194 (2009).

97 Santoro A, Rimassa L, Borbath I et al. Tivantinib for second-line treatment of advanced hepatocellular carcinoma. a randomised, placebo-controlled Phase 2 study. Lancet Oncol. 14(1), 55-63 (2013).

- Phase II study of c-MET tyrosine kinase inhibitor tivantinib after sorafenib failure in advanced HCC. 